\title{
Novel targeted puncture technique for percutaneous transforaminal endoscopic lumbar discectomy reduces $X$-ray exposure
}

\author{
YUEDONG ZENG ${ }^{1,2}$, JIE BAO $^{2}$, JIANCHENG SU $^{2}$, PINGXIAN TAN ${ }^{2}$, WEI XIE $^{2}$, ZHENG HUANG $^{3}$ and HONG XIA ${ }^{1}$ \\ ${ }^{1}$ Department of Spinal Surgery, General Hospital of Guangzhou Military Command of PLA, Affiliated with Southern \\ Medical University, Guangzhou, Guangdong 510000; Departments of ${ }^{2}$ Spinal Surgery and ${ }^{3}$ Clinical Laboratory, \\ Longgang District Central Hospital of Shenzhen, Shenzhen, Guangdong 518116, P.R. China
}

Received December 30, 2015; Accepted February 1, 2017

DOI: $10.3892 /$ etm.2017.4917

\begin{abstract}
The present study explored a method to reduce X-ray exposure dose and avoid targeted puncture complications in percutaneous transforaminal endoscopic lumbar discectomy (PTELD). A total of 66 patients with lumbar disc herniation were divided into two groups for a controlled study. In the experimental group, 31 patients were subjected to PTELD using a novel targeted puncture technique with application of a lumbar disc herniation target collimator. The remaining 35 patients in the control group were subjected to free-hand targeted puncture PTELD. The number of X-ray fluoroscopies performed intraoperatively, targeted puncture accuracy, visual analogue scale for surgical pain and Oswestry disability index of the two groups were statistically analyzed. The experimental and control groups exhibited a statistically significant difference in the number of $\mathrm{X}$-ray fluoroscopies required during the procedure $(\mathrm{P}<0.01)$. The number of successful first targeted punctures was $27(87.1 \%)$ in the experimental group and three $(8.6 \%)$ in the control group, indicating that the puncture accuracy was higher in the experimental group than in the control group. As for the pain response to outer sleeve insertion (local anesthetic injection through the guide sleeve), the experimental group had 25 mild cases $(80.6 \%)$, five moderate cases $(16.1 \%)$ and one severe care $(3.2 \%)$, whereas the control group had five mild cases (14.3\%), 19 moderate cases $(54.3 \%)$ and 11 severe cases $(31.4 \%)$. These results demonstrated that the overall pain response of the experimental group was milder than that of control group. Due to a larger puncture deviation,
\end{abstract}

Correspondence to: Professor Hong Xia, Department of Spinal Surgery, General Hospital of Guangzhou Military Command of PLA, Affiliated with Southern Medical University, 111 Liuhua Road, Guangzhou, Guangdong 510000, P.R. China

E-mail: gzxiahong2@126.com

Key words: transforaminal approach, percutaneous targeted puncture, puncture guiding device, X-ray exposure, lumbar discectomy the nerve root was touched by the puncture needle in 12 cases in the control group and resulted in one case of severe postoperative infection. In conclusion, the novel targeted puncture technique guided by a lumbar disc herniation target collimator outlined in the present study is able to markedly reduce X-ray exposure dose in PTELD and limit the surgical risk and pain experienced by patients. Mastering this novel puncture technique may aid those new to performing PTELD.

\section{Introduction}

Percutaneous transforaminal endoscopic lumbar discectomy (PTELD) is the most minimally invasive surgical method used to treat lumbar disc herniation (1-4). The procedure possesses the features and advantages of a minimally invasive treatment, including an extremely small incision, little bleeding, light damage and rapid recovery (5-8). PTELD has little effect on the structure of the spinal canal, does not affect spinal stability and forms only slight scar adhesions. PTELD is currently a central focus in spinal surgery research on minimally invasive treatments for lumbar disc herniation.

The first step of PTELD requires the puncture needle to percutaneously pass through the narrow intervertebral foramen, and the puncture needle tip enters into the location of lumbar disc herniation tissue that compresses the nerve root in the spinal canal (also known as the target spot). This process is referred to as percutaneous targeted puncture $(9,10)$. Following this initial step, guide wire is inserted through the puncture needle and a dilating sleeve is inserted under the guidance of the guide wire. The working sleeve is placed in the target spot to effectively remove lumbar disc herniation tissue under full endoscopy to relieve the nerve root from compression. At present, surgeons perform this puncture surgery free-hand, relying on their sense of touch and surgical experience. When conducting PTELD free-hand, multiple X-ray fluoroscopies are required to confirm the locations of the puncture needle tip and the sleeve's front end and to repetitively adjust the angle of the puncture needle. Therefore, both the operator and patient suffer from high doses of $\mathrm{X}$-ray radiation, which is extremely unfavorable for their health. According to a study by Zhou et al (11), the number of X-ray fluoroscopies performed 
for targeted puncture and sleeve insertion may be as high as 32 . Evidence has suggested that the duration of X-ray fluoroscopy may be 1.6-4.5 min, and the X-ray exposure dose for surgeons new to the technique may be even more (12). As X-ray exposure causes damage to the human body, there are few surgeons who are willing to learn this technique. Furthermore, free-hand targeted puncture may have large deviations, which may cause damage to important blood vessels, nerves, the intestinal canal and other tissues, resulting in severe adverse effects. For proficient surgeons with effective sense of touch and rich practical experience $(11,13)$, the free-hand percutaneous targeted puncture technique is able to achieve relatively high accuracy and reduced X-ray exposure. However, for beginners who are not yet proficient, have less effective sense of touch and poor practical experience, percutaneous targeted puncture is a difficulty they are required to overcome in order to master the PTELD technique.

In order to reduce the number of times and dose of X-ray exposure during free-hand targeted puncture and sleeve insertion in PTELD, as well as to increase targeted puncture accuracy and avoid puncture deviation, the present study developed a lumbar disc herniation target collimator and used a newly established methodology to guide the targeted puncture (14) (Fig. 1). This novel technique is able to accurately guide the puncture needle to percutaneously pass through the narrow intervertebral foramen in the first attempt, and enter the location of lumbar disc herniation in the spinal canal. The present controlled clinical study was conducted using the new targeted puncture technique with the application of the lumbar disc herniation target collimator.

\section{Materials and methods}

\section{Ethics statement}

Patients. Between January and September of 2014, 66 lumbar disc herniation patients were admitted and treated within the Department of Spinal Surgery, Longgang District Central Hospital of Shenzhen (Shenzhen, China). All patients gave their written informed consent prior to participating in the study. The present study was approved by the Ethics Committee of Longgang District Central Hospital of Shenzhen. Patients were divided into two groups based on their dates of birth: Patients whose date of birth was an even number were assigned to the experimental group and underwent PTELD using a novel targeted puncture technique with application of a lumbar disc herniation target collimator; and patients whose date of birth was an odd number were assigned to the control group and underwent free-hand targeted puncture PTELD. Inclusion criteria for the patients were as follows: Patients diagnosed with single-level lumbar disc herniation; and with the lumbar disc herniation tissue located inside the spinal canal. Exclusion criteria for the patients were as follows: Patients with L5/S1 lumbar disc herniation with the line between the peak points of bilateral iliac crests being higher than 1/3 of L5 vertebra; patients whose herniated lumbar disc was obviously unstable; patients with psychological factors; patients with hypertension; patients with coagulopathy, lung and brain diseases; and patients with low pain thresholds and intolerance to local anesthesia.

The experimental group contained 31 patients, including 20 males and 11 females aged from 25 to 50 years old, with a mean age of 37.5 years. The patients had suffered from lumbocrural pain for 1.5-48 months, with a mean of 14 months of pain. Their lumbocrural pain visual analogue scale (VAS) score was $6.5161 \pm 0.9957$ and Oswestry disability index (ODI) score was $65.1935 \pm 10.9436$. The herniated segment was L3/4 in two cases, L4/L5 in 24 cases and L5/S1 in five cases. The control group contained 35 patients, including 19 males and 16 females aged from 28 to 54 years old, with a mean age of 39.7 years. Control patients had suffered from lumbocrural pain for 2-48 months with a mean of 14.3 months of pain. Their lumbocrural pain VAS score was $6.6857 \pm 0.9632$ and ODI score was $65.7714 \pm 10.6470$. The herniated segment was L4/L5 in 27 cases and L5/S1 in eight cases. There was no significant difference in age, duration of lumbocrural pain, VAS score and ODI score between the two groups ( $\mathrm{P}>0.01)$, as exhibited in Table I.

All patients received lumbar anteroposterior and lateral $\mathrm{X}$-ray radiographs, lumbar over flexion-extension X-ray radiographs, as well as imageological examinations of lumbar computed tomography (CT) scans and lumbar magnetic resonance imaging (MRI). All surgeries were performed by the same surgeon and conversation with the patients was maintained during surgery. The surgeon was immediately informed when the patient exhibited lower limb or buttock pain, which indicated that the puncture needle tip had touched the nerve root. If this occurred, the puncture needle was immediately withdrawn to avoid aggravating or causing further damage to the nerves.

\section{Surgical methods}

Surgical method for the experimental group. First, the lumbar disc herniation tissue location (target spot) was determined from lumbar X-ray radiographs, CT and MRI images based on the downward displacement of lumbar disc herniation tissue exhibited on sagittal images of CT or MRI scans (Figs. 2 and 3). On lumbar anteroposterior X-ray radiographs, the cranial angle $\alpha$ of the lumbar disc's parallel line was measured on the coronal plane and the value of angle $\alpha$ was the angle that was required to be set for the $\alpha$ angle locator (Fig. 4). On cross-sectional CT or MRI images, line AO for the optimum puncture path from the back side of the waist to the target spot was customized (the path line must not pass the front edge of the iliocostalis, intestinal canal or other tissues that may be injured), and the $\beta$ angle between the puncture path line $\mathrm{AO}$ and the coronal plane was measured. The value of angle $\beta$ was the angle that was required to be set for the $\beta$ angle locator (Fig. 5). On the lumbar overflexion X-ray radiographs, the vertical projection point (B) of the target spot was marked on the edge of the skin of the waist, and the distance (d) from the target spot $(\mathrm{O})$ in the spinal canal to its skin projection point (B) was measured (Fig. 6). According to the orthogonal trigonometric function relationship, the distance between the circle center of the $\alpha$ angle locator and the circle center $(\mathrm{E})$ of the $\beta$ angle locator was calculated as $\mathrm{d} 1=\mathrm{d} / \operatorname{tg} \beta$ and the distance between the target spot $(\mathrm{O})$ and the circle center $(\mathrm{E})$ of the $\beta$ angle locator was calculated as $\mathrm{d} 2=\mathrm{d} / \sin \beta$.

The patient was asked to lie in the prostrate position on the operating table, and the position was adjusted to allow for lumbar overflexion so that the waist plane was parallel to the floor. The patient was kept immobilized. Bony structures of the subcutaneous lumbar spine were touched and lines between 
Table I. General characteristics of patients in the experimental and control group before surgery.

\begin{tabular}{lccr}
\hline Characteristics & Experimental group & Control group & P-value \\
\hline Sex, male/female & $20 / 11$ & $19 / 16$ & \\
Age, years & $37.4839 \pm 7.6587$ & $39.7143 \pm 7.3707$ & 0.233 \\
Duration of lumbocrural pain, months & $13.9839 \pm 11.7022$ & $14.3143 \pm 11.2819$ & 0.907 \\
Preoperative VAS & $6.5161 \pm 0.9957$ & $6.6857 \pm 0.9632$ & 0.485 \\
Preoperative ODI & $65.1935 \pm 10.9436$ & $65.7714 \pm 10.6467$ & 0.829 \\
Herniated lumbar disc segment (no. of patients) & L3/4 (2), L4/5 (24), L5/S1 (5) & L4/5 (27), L5/S1 (8) &
\end{tabular}

Data are presented as the mean \pm standard deviation. VAS, visual analogue scale; ODI, Oswestry disability index.

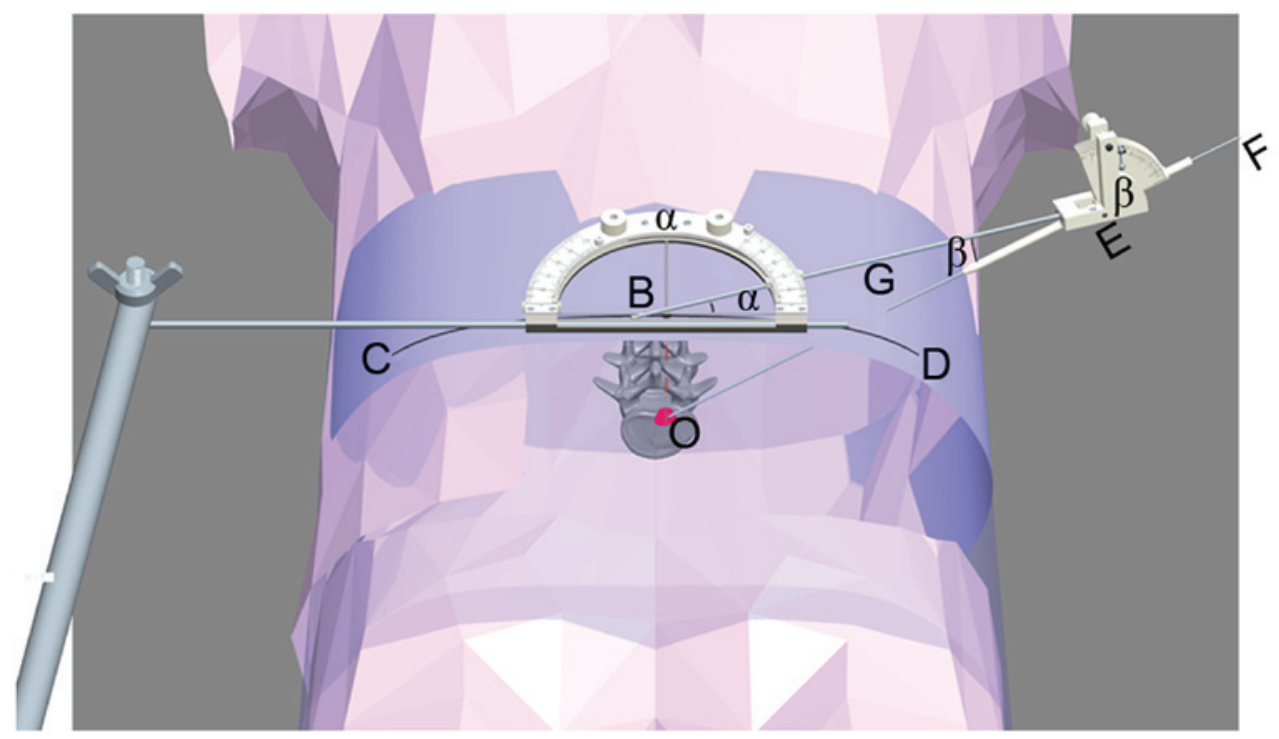

Figure 1. Structure of lumbar disc herniation target collimator and schematic diagram of the principle of guided targeted puncture. $\alpha$ represents the $\alpha$ angle locator, which has a semicircular structure. $\beta$ represents the $\beta$ angle locator, which has a quarter-circular structure. $\alpha$ and $\beta$ angle locators are connected by a telescopic rod and together constitute the target collimator. Point $\mathrm{O}$ is the lumbar disc herniation tissue (target spot). Point B is the vertical projection of the target spot on the skin of the waist. Point $\mathrm{G}$ is the puncture spot where the puncture needle passes through the skin. Point $\mathrm{E}$ is the circle center of the $\beta$ angle locator. Point $\mathrm{F}$ is the tail end of the puncture needle. Line $\mathrm{OB}$ is the distance between the target spot and its skin vertical projection point. Line $\mathrm{CD}$ is the parallel line on the skin to the lumbar disc passing through point B. Angle $\alpha$ is the included angle between the telescopic angle and the $\alpha$ angle locator's diameter; the $\alpha$ angle locator's diameter overlaps with line $\mathrm{CD}$. Angle $\beta$ is the included angle between the telescopic rod and the puncture needle.

lumbar spines were marked. A roentgen opaque positioning grid was flatly placed over the skin of the waist, and the first lumbar anteroposterior X-ray fluoroscopy was performed using a mobile C-arm X-ray machine. Following this, the lumbar disc's parallel line $(\mathrm{CD})$ and the vertical projection point $(\mathrm{B})$ of the target spot were marked on the skin. Disinfection was frequently performed on the skin surface around the surgical area and an aseptic towel was subsequently placed over the skin. The $\alpha$ angle locator was horizontally placed on the skin of the waist so that the circle center of the $\alpha$ angle locator was on the vertical projection point (B) and the diameter of the $\alpha$ angle locator was on the lumbar disc's parallel line (CD). The angle of the $\alpha$ angle locator was adjusted to the measured $\alpha$ value by rotating its rotary knob, and the length of the connecting telescopic rod was adjusted so that the distance between the circle center (B) of the $\alpha$ angle locator and circle center (E) of the $\beta$ angle locator was equal to d1. The $\beta$ angle locator was then kept in vertical balance and adjusted to the measured $\beta$ value by rotating its rotary knob. The puncture needle was subsequently inserted into the guide wire and slowly pushed through skin-muscle tissues to pass through the narrow intervertebral foramen until it entered into the target spot $(\mathrm{O})$ inside the spinal canal. During puncturing, the distance between the circle center $(\mathrm{E})$ of the $\beta$ angle locator and the puncture needle tip $F$ (the EF distance was presented as $\mathrm{d} 3$ ) was measured. When the total length of the puncture needle subtracted by $\mathrm{d} 3$ equaled $\mathrm{d} 2$, it indicated that the puncture needle tip had reached the target spot. A mobile C-arm X-ray machine (Mobile X-ray Image System; ARCADIS Orbic/orbic 3D; Siemens AG, Muenchen, Germany) was used for the second lumbar anteroposterior fluoroscopy and the third lumbar lateral fluoroscopy to confirm the puncture needle tip was at the target spot. The collimator was then removed and the distance on the puncture needle between the puncture point $(\mathrm{G})$ and the point $\mathrm{F}$ (presented as $\mathrm{d} 4$ ) was measured. The depth from point $\mathrm{G}$ to target spot $\mathrm{O}$ was obtained by subtracting $\mathrm{d} 4$ from the total length of the puncture needle (Fig. 1). 


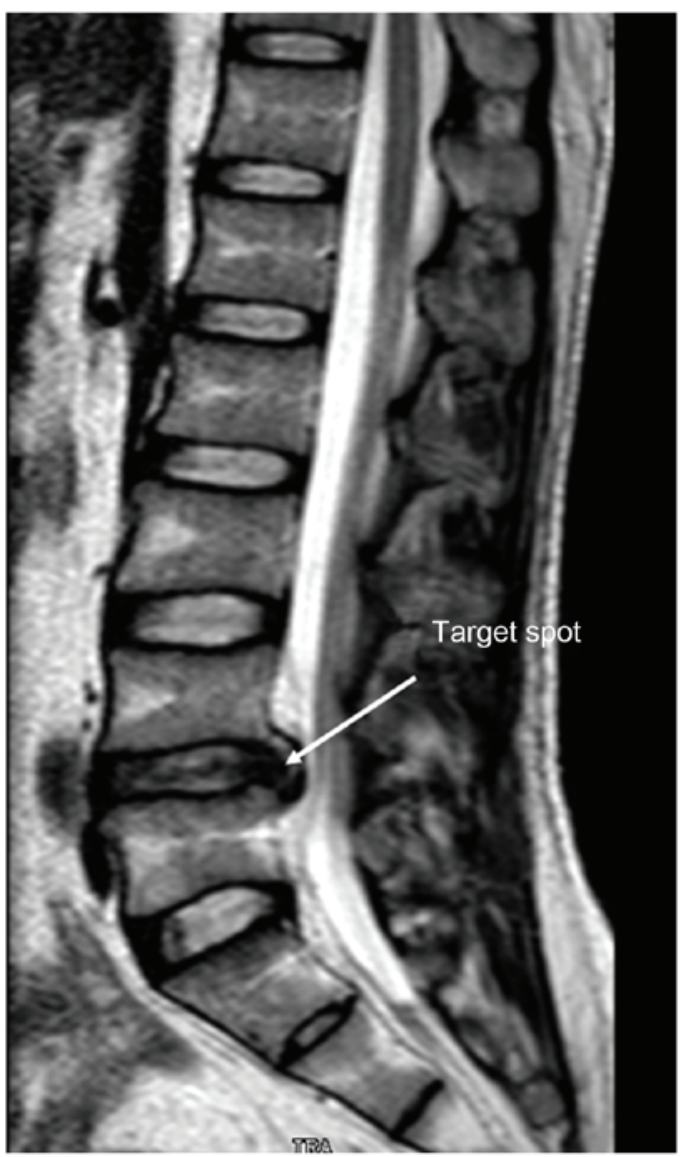

Figure 2. Sagittal plane of lumbar magnetic resonance image. The arrow indicates the location of herniated lumbar disc tissue (target spot).

Local subcutaneous infiltration anesthesia was performed for the puncture using $2 \%$ lidocaine. The puncture needle's stylet was removed and guide wire was inserted. Subsequently, the puncture needle was withdrawn and a $\sim 1 \mathrm{~cm}$ long horizontal incision was made at the puncture point. Expanding sleeves were inserted step-by-step under the guidance of guide wire until the working sleeve was inserted into the correct location. During sleeve insertion, the sleeve length exposed outside of the skin was measured, and the sleeve length inside the body was calculated by subtracting the outside sleeve length from the total sleeve length. When the length of the sleeve in the body equaled the value of $\mathrm{d} 4$, the front end of the sleeve had reached the target spot. The mobile $\mathrm{C}$-arm X-ray machine was used for the fourth lumbar anteroposterior fluoroscopy and the fifth lumbar lateral fluoroscopy to confirm the sleeve's front end was at the target spot. A full endoscope was placed inside the working sleeve and the nerve-compressing lumbar disc herniation tissue was directly removed under the monitoring of a video camera. The nerve root was probed and loosened and bipolar radiofrequency ablation (to decompress the intervertebral disc) and angioplasty were performed.

Surgical method for the control group. The surgical method for the control group was conducted according to previous research $(11,13,15)$. The patient was asked to lie in the prostrate position on the operating table, and the position was adjusted to allow for lumbar overflexion so that the waist

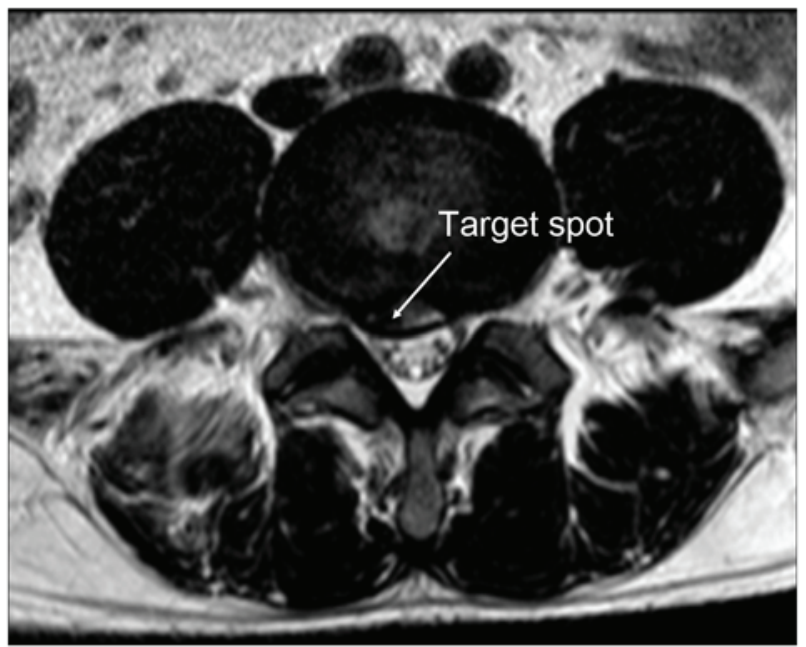

Figure 3. Cross-sectional plane of lumbar magnetic resonance image. The arrow indicates the location of herniated lumbar disc tissue (target spot).

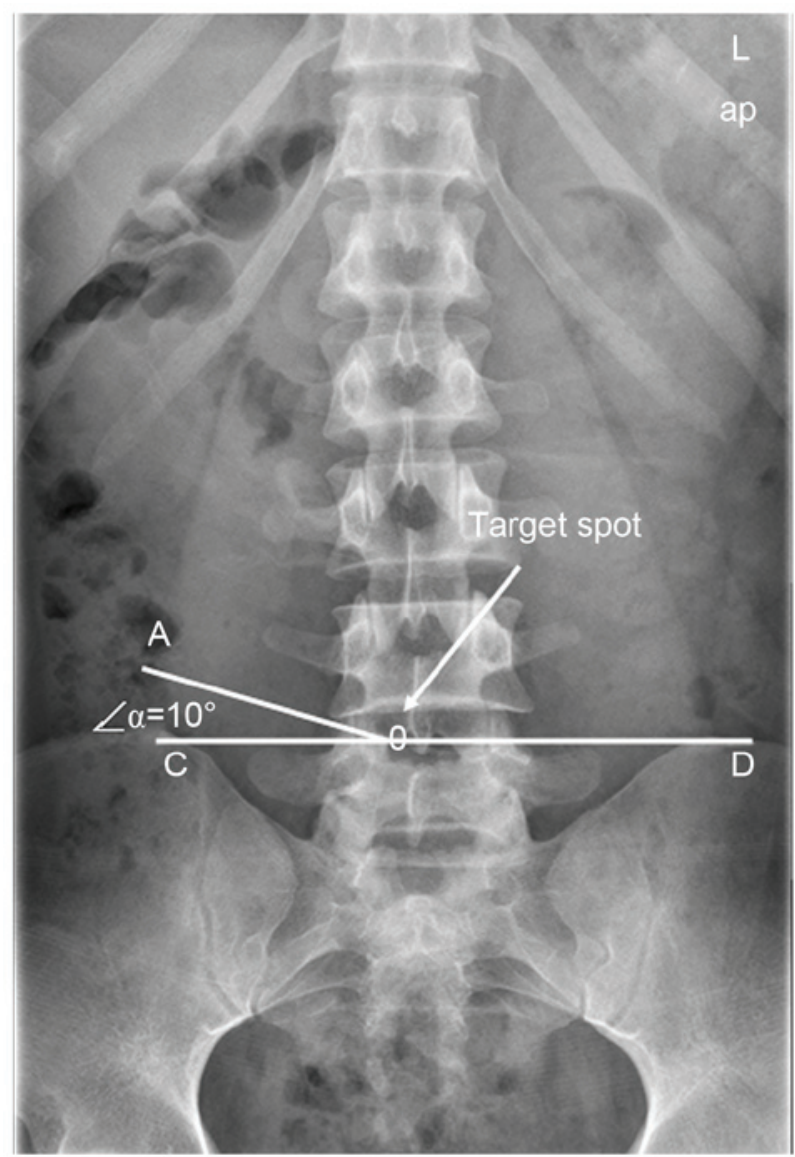

Figure 4. Lumbar anteroposterior X-ray radiograph. Point O on the coronal plane is the location of target spot. Line $\mathrm{CD}$, parallel line from the skin to lumber disc; Line AO, optimum percutaneous path line to the target spot; $\alpha$, the included angle between line $\mathrm{AO}$ and $\mathrm{CD} ; \mathrm{L}$, left of the spine; lat, lateral position; ap, lumbar anteroposterior X-ray radiograph.

plane was parallel to the floor. The center line of the lumbar vertebrae and a parallel line passing through the upper margin of lumbar disc were marked under lumbar anteroposterior fluoroscopy using a mobile C-arm X-ray machine. A lateral line through the upper posterior margin of the lower vertebrae 


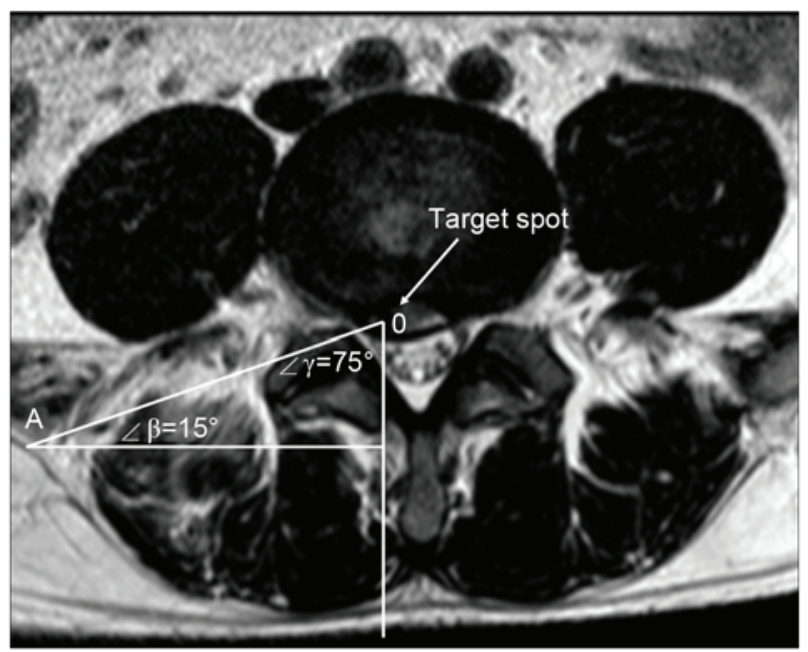

Figure 5. Cross-sectional plane of lumbar magnetic resonance image. Line AO is the optimum percutaneous puncture path line to the target spot; $<\beta$ is the included angle between $\mathrm{AO}$ and the coronal plane and $<\gamma$ is the included angle between $\mathrm{AO}$ and the sagittal plane.

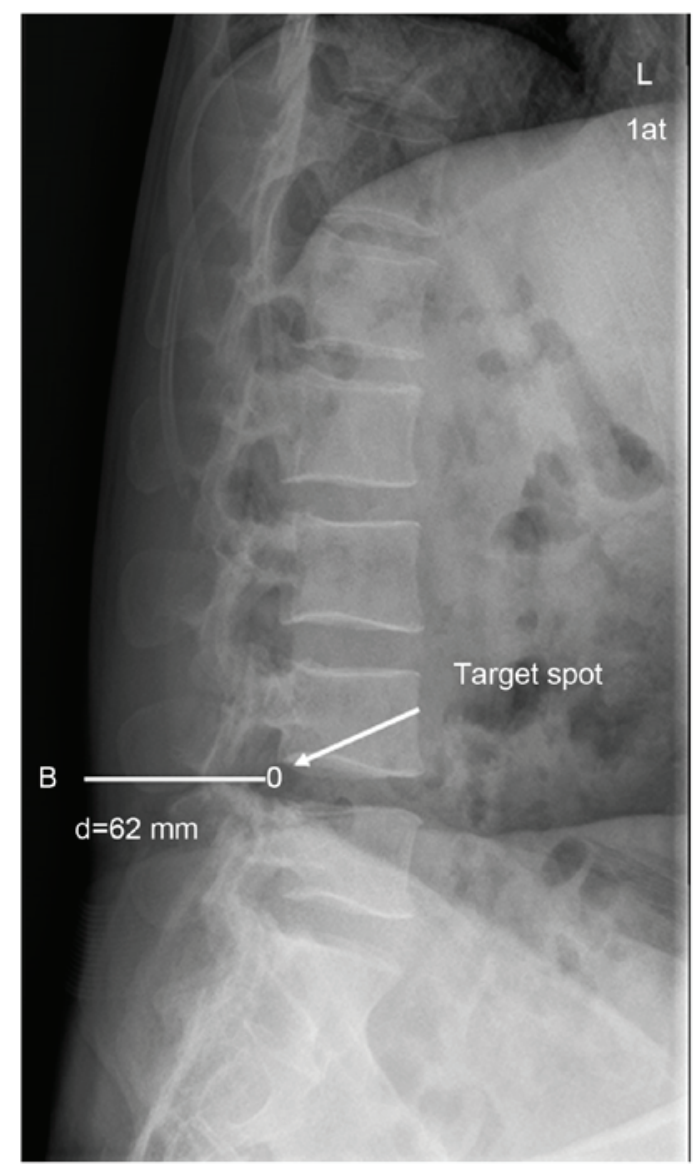

Figure 6. Lumbar overflexion $\mathrm{X}$-ray radiograph. Point $\mathrm{O}$ is the location of the target spot, point $\mathrm{B}$ is the vertical projection of target point $\mathrm{O}$ on the plane of the skin of the waist and line BO is the distance between target spot $\mathrm{O}$ and its projection $\mathrm{B}$, which is equal to $\mathrm{d}$. $\mathrm{L}$, left of the spine; lat, lateral position.

following the direction of intervertebral tilt under lumbar lateral fluoroscopy was also marked. The intersection of the lateral line and the parallel line passing through the upper margin of the lumbar disc represented the puncture point. For the L5/S1 lumbar disc, the connecting line between the peak points of iliac crests and the parallel line passing through the upper margin of the L5/S1 lumbar disc were marked under lumbar anteroposterior fluoroscopy. A lateral line from the superior articular process of S1 to the upper posterior margin of S1 was also marked under lateral fluoroscopy. The intersection of the lateral line and the connecting line between the peak points of iliac crests represented the puncture point. The puncture was performed from the upper posterior margin of $\mathrm{S} 1$ at an angle between $30-40^{\circ}$ for L4/L5 lumbar disc abduction, and between $40-50^{\circ}$ for L5/S1 lumbar disc abduction.

Disinfection was frequently performed on the surface of the skin around the surgical area, and an aseptic towel was placed over the skin. Local anesthesia was performed for the puncture point using 2-3 ml lidocaine (1\%; v/v). Under lumbar anteroposterior and lateral X-ray fluoroscopy and following the marked line, the puncture needle was pushed free-hand through skin-muscle tissue and the front edge of the superior articular process to enter into the target spot in the spinal canal. The guide wire was subsequently inserted and the puncture needle was withdrawn. A $\sim 1 \mathrm{~cm}$ long horizontal incision was made at the puncture point. The expanding sleeves were inserted step-by-step under the guidance of guide wire and, in case there were any bony obstructions of the articular process, a serrated reamer was inserted to remove some of the bony substance from the lateral margin of the superior articular process. Subsequently, the working sleeve was inserted. During puncturing and sleeve insertion, the location of the puncture needle tip and the sleeve's front end were confirmed using multiple X-ray fluoroscopies. A full endoscope was placed inside the working sleeve and the nerve-compressing lumbar disc herniation tissue was directly removed under the monitoring of a video camera (Stryker 1288 HD Endoscopic Camera; Stryker, Kalamazoo, MI, USA). The nerve root was then probed and loosened and bipolar radiofrequency ablation decompression and angioplasty of the lumbar disc's annular gap were performed.

Observation method. The number of X-ray fluoroscopies performed during puncturing and sleeve insertion was recorded and the targeted puncture accuracy was observed. The degree of the patient's pain response during sleeve insertion was assessed and categorized into mild, moderate and severe. Mild pain indicated that the patient did not exhibit any reactive body movement to pain, with blood pressure $<140 \mathrm{mmHg}$, respiration rate $<22 \mathrm{~min}^{-1}$ and heart rate $<100 \mathrm{~min}^{-1}$. Moderate pain indicated that the patient exhibited slight reactive body movement to pain, with blood pressure $<140 \mathrm{mmHg}$, respiration rate $<22 \mathrm{~min}^{-1}$ and heart rate $<100 \mathrm{~min}^{-1}$. Severe pain indicated that the patient shouted with both hands fisted, the lumbar muscle was noticeably tense and obvious reactive body movement to pain was exhibited, with blood pressure $>140 \mathrm{mmHg}$, respiration rate $>22 \mathrm{~min}^{-1}$ and heart rate $>100 \mathrm{~min}^{-1}$. The surgical effect was evaluated according to VAS and ODI scores.

Statistical analysis. Statistical analysis was performed using SPSS v.21.0 (IBM SPSS, Armonk, NY, USA). Results were statistically analyzed using the Student's t-tests Data are presented as the mean \pm standard deviation). $\mathrm{P}<0.01$ was considered to indicate a statistically significant difference. 
Table II. Number of intraoperative X-ray fluoroscopies performed in the experimental and control groups.

\begin{tabular}{lccr}
\hline X-ray fluoroscopy type & Experimental group & Control group & P-value \\
\hline Target puncture & $3.2581 \pm 0.6816$ & $14.2857 \pm 7.1190$ & $<0.01$ \\
Sleeve insertion & $2.1935 \pm 0.6011$ & $19.2857 \pm 4.9620$ & $<0.01$ \\
Total & $5.4516 \pm 0.8500$ & $33.5714 \pm 8.7356$ & $<0.01$
\end{tabular}

Data are presented as the mean \pm standard deviation.

Table III. Number of targeted puncture attempts required in the experimental $(n=31)$ and control $(n=35)$ groups.

\begin{tabular}{lccccccccc}
\hline & \multicolumn{7}{c}{ No. of puncture attempts } \\
\cline { 2 - 9 } Group & 1 & 2 & 3 & 4 & 5 & 6 & 8 & 9 \\
\hline Experimental & 27 & 4 & 0 & 0 & 0 & 0 & 0 & 0 \\
Control & 3 & 2 & 6 & 7 & 7 & 2 & 2 & 1 \\
\hline
\end{tabular}

\section{Results}

PTELD using a lumbar disc herniation target collimator decreases the number of $X$-ray fluoroscopies required and increases the accuracy of initial puncture. The number of $\mathrm{X}$-ray fluoroscopies performed in the experimental group (5.4516 \pm 0.8501$)$ was significantly lower than that of the control group (33.5714 $\pm 8.7356 ; \mathrm{P}<0.01$; Table II). As for puncture accuracy, the experimental group had 27 successful cases $(87.1 \%)$ in the first attempt and 4 successful cases (12.9\%) in the second attempt. The control group had 3 successful cases $(8.6 \%)$ in the first attempt, 2 successful cases $(5.7 \%)$ in the second attempt, 6 successful cases $(17.1 \%)$ in the third attempt, 7 successful cases $(20.0 \%)$ in the fourth attempt, 7 successful cases $(20.0 \%)$ in the fifth attempt, 2 successful cases $(5.7 \%)$ in the sixth attempt, 2 successful cases (5.7\%) in the eighth attempt, 1 successful case $(2.9 \%)$ in the ninth attempt and 5 successful cases $(14.3 \%)$ in the tenth attempt or more. The puncture accuracy of the experimental group was markedly higher than that of the control group (Table III).

PTELD using a lumbar disc herniation target collimator decreases pain experienced by patients. As for patient's pain response during sleeve insertion under local anesthesia, the experimental group had 25 mild cases (80.6\%), 5 moderate cases $(16.1 \%)$ and 1 severe case (3.2\%).The control group had 5 mild cases (14.3\%), 19 moderate cases (54.3\%) and 11 severe cases $(31.4 \%)$. The overall pain response of the experimental group was significantly milder than that of the control group $(\mathrm{P}<0.01$; Fig. 7$)$.

A total of 5 patients in the control group experienced lower limb radiating pain during puncturing and seven cases experienced buttock pain. The patients informed the surgeon when they experienced pain and puncturing was stopped immediately. The puncture needle was withdrawn and re-punctured following adjustment of the needle angle. No symptoms of nerve root damage were observed. One case in the control group exhibited repetitive hyperpyrexia on the second day after the surgery and

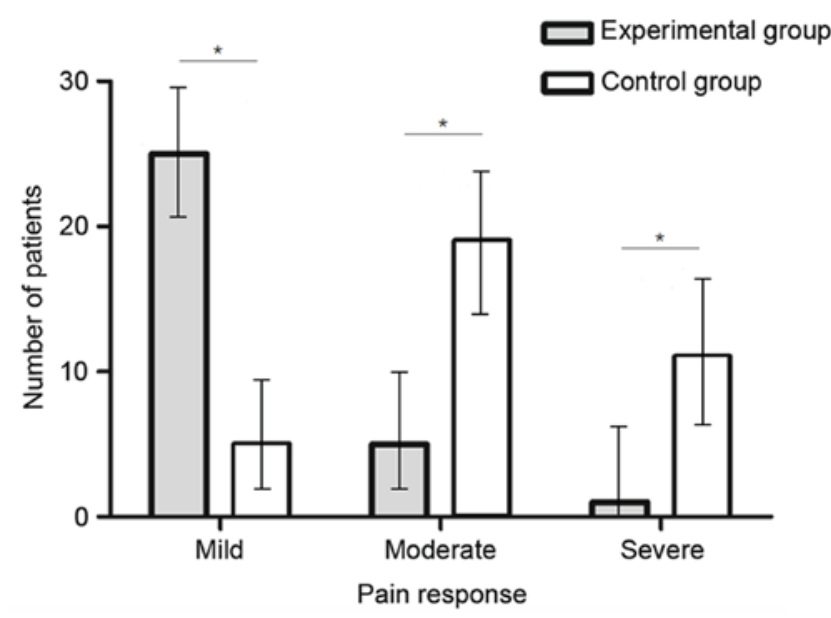

Figure 7. Degree of pain response exhibited during sleeve insertion in the experimental and control groups. ${ }^{*} \mathrm{P}<0.01$ vs. the control group.

this lasted for 1 week. Examination results of blood procalcitonin for this patient was $7.51 \mathrm{ng} / \mathrm{ml}$ (the normal range is $>0.05 \mathrm{ng} / \mathrm{ml}$ ). The patient subsequently exhibited secondary pleural effusion and ascites, jaundice and impaired liver function. Treatment using common antibiotics was ineffective, and lumber MRI performed 1 week after surgery demonstrated that infection foci were present in the surgical segment of the lumbar vertebrae and the spinal canal. Through multidisciplinary consultation and discussion, the patient was diagnosed with bloodstream infection, which was suspected to be caused by the large deviation of free-hand puncture that resulted in the puncture needle entering the patient's intestinal canal and being withdrawn and reused for puncture. Thus intestinal bacteria was carried into the lumbar vertebra, resulting in severe infection. Fortunately, the patient's condition was effectively controlled through the administration of the antibiotic imipenem cilastin sodium $(0.5 \mathrm{~g}$ once every $6 \mathrm{~h}$ for 2 weeks; Merck Sharp \& Dohme Corp., Elkton, VA, USA) via an intravenous drip. Both groups experienced no nerve injury, major blood vessel damage or dural sac tearing complications. 
Table IV. Comparison between postoperative lumbocrural pain VAS and lumbar ODI in the experimental and control groups.

\begin{tabular}{lcrr}
\hline Measure of pain & Experimental group & Control group & P-value \\
\hline Postoperative VAS & $1.2581 \pm 0.9298$ & $1.4571 \pm 1.0939$ & 0.427 \\
Postoperative ODI (\%) & $16.1935 \pm 8.4437$ & $18.0571 \pm 9.1071$ & 0.394 \\
\hline
\end{tabular}

Data are presented as the mean \pm standard deviation. VAS, visual analogue scale; ODI, Oswestry disability index.

Postoperative follow-up was conducted for 9-15 months, with a mean of 12.6 months. VAS and ODI scores of the two groups were not significantly different (P>0.01; Table IV).

\section{Discussion}

Lumbar intervertebral foramina are narrow and usually obstructed by the articular process and the transverse process at the back. Important tissues pass through the lumbar intervertebral foramina, including blood vessels, the intestinal canal and nerve roots. The majority of intervertebral disc herniation tissues are located inside the spinal canal and intervertebral foramina and often exhibit downward displacement. Upward displacements are only observed in rare cases. When performing PTELD free-hand, the puncture needle passes through the narrow intervertebral foramen and enters the spinal canal to reach the point of intervertebral disc herniation. Surgeons must rely on their hand's sense of touch and practical experience and, therefore, in the majority of cases, the puncture cannot be successfully conducted on the first attempt. X-ray fluoroscopy is required to be performed repeatedly in order to confirm the location of the puncture needle tip. In the case of an undesired puncture needle tip location, it is necessary for the needle angle to be adjusted or for the puncture point location to be altered. Repeated X-ray fluoroscopies result in increased X-ray exposure and once X-ray exposure exceeds the occupational exposure limit (16) it will cause radioactive damage to the human body.

In the experimental group of the present study, the depth from the puncture point to the target spot had been measured in advance and the length of the sleeve's in-skin part and its location were estimated by measuring the length of the exposed part of the sleeve outside of the skin during sleeve insertion. This meant that repetitive X-ray fluoroscopy was not necessary to confirm the location of the sleeve's front end. In this way, X-ray exposure dose may be effectively reduced and radioactive damage to the surgeon and patient may be avoided. During free-hand targeted puncture, involuntarily shaking of the surgeon's hands may cause deviation of the puncture needle from the target spot. A large deviation may injure important blood vessels, nerves, the intestinal tract and other tissues, and even cause severe adverse outcomes. In the control group of the present study, the puncture deviation was relatively large in 12 cases, in which the puncture needle touched nerve roots and induced lower limb and buttock pain. However, the surgeon communicated with the patients throughout the procedure and the puncture needles were withdrawn as soon as the patient experienced any pain. This meant that accidental puncture injury to the nerve roots was limited. In 1 case of the control group, the patient had a relatively smaller body shape compared with the other patients and free-hand puncture had a large deviation. The puncture needle entered the intestinal canal and, after withdrawal and re-puncturing, carried intestinal bacteria into the lumber vertebra after the needle angle had been adjusted. This resulted in a severe bloodstream infection. Fortunately, the infection was effectively controlled following antibiotic treatment. In the experimental group, a collimator was used to guide the puncture needle during the procedure. Once the puncture angle and depth were preset, the puncture needle was slowly inserted along the guide tube without shaking, thus effectively avoiding injury to important tissues and reducing the surgical risk. Common complications of PTELD are nerve injury and cerebrospinal fluid leak (1-3). The incidences of hematoma and infection are relatively low, at 0.97 (17) and $0.12 \%$ (18), respectively. It is evident that large puncture deviation may injure the blood vessels and cause hematoma (17); however, whether nerve injury, cerebrospinal fluid leak and infection are associated with puncture injury has not been reported.

The intervertebral disc herniation target collimator developed in the present study performs 3D target collimation based on the orthogonal trigonometric function relationship between one distance and two angles. The collimator has a simple structure and is easy to operate. Once the distance from the target spot of lumbar disc herniation tissue to the spot's vertical projection point on the lumbar plane, and the two puncture angles between the puncture path line and the sagittal plane and cross-section plane are determined, the puncture depth may be calculated by the orthogonal trigonometric function relationship. The calculation method is scientific and has high puncture accuracy. Contrastingly, other intervertebral disc puncture locators (19-24) have poor puncture accuracy and no scientific puncture methodology and only guide the puncture needle into the intervertebral disc. Such methods are only suitable for rough positioning and puncture, and are not able to reach the extremely small locations of intervertebral disc herniation tissue in the spinal canal. The discectomy performed following puncture guidance by such intervertebral disc locators is only able to achieve indirect decompression of nerve roots, and is far from meeting the requirement of high accuracy in targeted puncture of transforaminal endoscopic discectomy (25). Furthermore, waist size varies among patients, and the collimator developed in the present study is suitable for each individual patient due to its unique design of a telescopic rod with adjustable length.

Individual patients with lumbar disc herniation suffer from varying degrees of kyphoscoliosis and rotation deformities, lumbar disc herniation tissue location varies from patient to 
patient and lumbar anatomical variation may exist. Therefore, when applying a posterolateral percutaneous transforaminal approach, each patient has a different puncture point location, angle and surgical path, highlighting the importance of a personalized surgical approach. It is necessary for the puncture path and surgical approach to be personally customized prior to surgery, thus selecting the best personalized surgical approach is a surgeon's primary concern. The most effective surgical approach, which may be designed preoperatively based on lumbar MRI or CT images, is required to avoid damage to bone, nerves, blood vessels, the intestinal tract and to muscle tissue.

The targeted puncture methodology designed in the present study meets the requirements of a personalized surgical approach. The puncture path is designed on lumbar MRI or CT cross-sectional images. The punctures performed in the experimental group had large abduction angles of $65-80^{\circ}$. The puncture point was relatively far from the mid-spinal line, at a distance of 12-15 cm, making it possible to effectively avoid bone substance obstruction of the articular process. This meant that a reamer was not required to remove bone substances and the patient experienced minimal pain during sleeve insertion. In comparison, in order to avoid large puncture deviation that may injure the vessels, nerves, intestinal canal and other tissues, free-hand puncture performed in the control group had smaller abduction angles of approximately $30-50^{\circ}$. The puncture point was relatively close to the mid-spinal line at a distance of 9-12 cm, which meant encounters with bony obstructions of the superior articular process were possible. Thus, it was necessary for some bone substances to be removed to enlarge the intervertebral foramen so that the sleeve was able to enter into the spinal canal. The patients in the control group exhibited the highest number of severe pain responses during sleeve insertion. Patients in the experimental group exhibited milder pain responses during sleeve insertion and were overall more relaxed throughout the surgical process compared with the control group.

Certain errors may be made when measuring the distance between the target spot and its vertical projection on the lumbar plane. The reason why the present study chose to perform the measurement on the lumbar overflexion X-ray radiograph is because the patient's prostrate position on the operating table is similar to lumbar overflexion. The vertical projection of the target spot on the lumbar plane was within the $1-2 \mathrm{~cm}$ radius of the spinous process under the majority of circumstances, thus it was not possible to use CT images and MRI to measure the distance between the target spot and its vertical projection on the skin. As the skin at the spinous process of the mid-spinal line is sunken while the erector spinae uplifts, the measured distance is smaller than the distance on the lumbar plane. If the patient is not in a suitable position when taking the lumbar overflexion X-ray radiograph prior to surgery, it may increase the measurement error, which would subsequently need to be corrected. If the puncture needle tip is found to be deviated from the target spot by X-ray fluoroscopy, it may be fixed by simply fine-adjusting the $\beta$ angle locator of the collimator. As the PTELD technique has a long and steep learning curve (26-29) and high technical difficulty, it is not easy to master $(30,31)$. Inexperienced surgeons are not necessarily able to accurately puncture the needle onto the target spot in the spinal canal even after repeated adjustments of the puncture needle angle and location, thus they are unable to effectively remove nerve-compressing intervertebral disc herniation tissues. Therefore, it is only possible to perform indirect nerve root decompression and the surgical effect will reduce due to incomplete nerve decompression. The collimator and the novel methodology of guided puncture presented in the present study is able to theoretically shorten the learning curve; however, this was not considered in the present study and will be the focus of future research.

The present study has adequately demonstrated that using the novel collimator-guided targeted puncture technique is able to effectively reduce the exposure of the human body to high $\mathrm{X}$-ray doses, as well as limit a patient's pain and the surgical risk. Renowned experts proficient in surgery with steady hands, effective sense of touch and rich practical experience are able to achieve high accuracy and reduce $\mathrm{X}$-ray exposure when performing free-hand percutaneous targeted puncture; however, for surgeons without proficient surgical skills and surgical experience, using the new collimator-guided targeted puncture technique may be more effective.

\section{Acknowledgements}

The present study was financially supported by the Shenzhen Knowledge Innovation Program (grant no. 20150225094534).

\section{References}

1. Jasper GP, Francisco GM and Telfeian A: Outpatient, awake, ultra-minimally invasive endoscopic treatment of lumbar disc herniations. R I Med J (2013) 97: 47-49, 2014

2. Sencer A, Yorukoglu AG, Akcakaya MO, Aras Y, Aydoseli A Boyali O, Sencan F, Sabanci PA, Gomleksiz C, Imer M, et al: Fully endoscopic interlaminar and transforaminal lumbar discectomy: Short-term clinical results of 163 surgically treated patients. World Neurosurg 82: 884-890, 2014

3. Choi KC, Kim JS, Ryu KS, Kang BU, Ahn Y and Lee SH: Percutaneous endoscopic lumbar discectomy for L5-S1 disc herniation: Transforaminal versus interlaminar approach. Pain Physician 16: 547-556, 2013.

4. Xu Z, Shi L, Chu L, Chen L, Ke Z and Deng Z: Comparison of percutaneous endoscopic via interlaminar and transforaminal approach for lumbar disc herniation. J Spinal Surg 11: 97-100, 2013 (In Chinese).

5. Ruetten S, Komp M, Merk H and Godolias G: Recurrent lumbar disc herniation after conventional discectomy: A prospective, randomized study comparing full-endoscopic interlaminar and transforaminal versus microsurgical revision. J Spinal Disord Tech 22: 122-129, 2009.

6. Hsu SK: Percutaneous endoscopic surgery to manage lumbar spine disease long time result report. Chin J Spine and Spinal Cord: 330-335, 2009 (In Chinese).

7. Birkenmaier C, Komp M, Leu HF, Wegener B and Ruetten S: The current state of endoscopic disc surgery: Review of controlled studies comparing full-endoscopic procedures for disc herniations to standard procedures. Pain Physician 16: 335-344, 2013.

8. Ruetten S, Komp M, Merk H and Godolias G: Full-endoscopic interlaminar and transforaminal lumbar discectomy versus conventional microsurgical technique: A prospective, randomized, controlled study. Spine (Phila Pa 1976) 33: 931-939, 2008.

9. Li CQ, Zhou Y, Wang J, Chu T, Zhang Z, Zheng W, Luo G: Minimally invasive targeted percutaneous endoscopic lumbar discectomy for lumbar disc herniation. Chin J Spine Spinal Cord 23: 193-197, 2013 (In Chinese).

10. Kim MJ, Lee SH, Jung ES, Son BG, Choi ES, Shin JH, Sung JK and Chi YC: Targeted percutaneous transforaminal endoscopic diskectomy in 295 patients: Comparison with results of microscopic diskectomy. Surg Neurol 68: 623-631, 2007. 
11. Zhou Y, Li CQ, Wang J, Zhang Z, Chu T, Pan Y, Zheng W and Luo G: Technique skill and clinical choices of the YESS and TESSYS in percutaneous transforaminal endoscopic discectomy for lumbar disc herniations. China J Orthop: 225-231, 2010 (In Chinese).

12. Ahn Y, Kim CH, Lee JH, Lee SH and Kim JS: Radiation exposure to the surgeon during percutaneous endoscopic lumbar discectomy: A prospective study. Spine (Phila Pa 1976) 38 617-625, 2013.

13. Hoogland T,SchubertM,MiklitzB and Ramirez A:Transforaminal posterolateral endoscopic discectomy with or without the combination of a low-dose chymopapain: A prospective randomized study in 280 consecutive cases. Spine (Phila Pa 1976) 31: E890-E897, 2006.

14. Zeng YD: Targeted guide apparatus of lumbar disc herniation by gear regulating. CN Patent 203724223U. Filed February 24, 2014; issued July 23, 2014.

15. Ahn Y, Oh HK, Kim H, Lee SH and Lee HN: Percutaneous endoscopic lumbar foraminotomy: An advanced surgical technique and clinical outcomes. Neurosurgery 75: 124-133, 2014

16. National Council on Radiation Protection and Measurements Recommendation on limits for exposure of ionizing radiation NCRP, Report no 91, Bethesda, MD, USA, 1987.

17. Ahn Y, Kim JU, Lee BH, Lee SH, Park JD, Hong DH and Lee JH: Postoperative retroperitoneal hematoma following transforaminal percutaneous endoscopic lumbar discectomy. J Neurosurg Spine 10: 595-602, 2009.

18. Ahn Y and Lee SH: Postoperative spondylodistic following transforaminal percutaneous endoscopic lumbar discectomy: Clinical characteristics and preventive strategies. Br J Neurosurg 26: 482-486, 2012.

19. Chen FQ: A lumbar disc puncturing assist device. CN Patent 203122556U. Filed March 21, 2013; issued August 14, 2013.

20. Wang H: Simple locator for lumbar puncture. CN Patent 202376229U. Filed December 15, 2011; issued August 15, 2012.

21. Xiong HH, Li XR and Peng JL: Automatic intervertebral disc puncturing system. CN Patent 201888837U. Filed November 16, 2010; issued July 06, 2011.
22. Zheng MH: Positioning device of percutaneous lumbar intervertebral disc operation path. CN Patent 101828966B. Filed April 28, 2010; issued February 01, 2012.

23. Huang X. Intervertebral disk puncture position finder. CN Patent 201519198U. Filed October 30, 2009; issued July 07, 2010.

24. Zhang GY, Guo YJ and Su WJ: Lumbar vertebral disc puncture locator. CN Patent 201375570. Filed April 13, 2009; issued January 06, 2010.

25. Yin HP, Li Sw, Du ZC, et al: Percutaneous lumbar disctomy associated with coblation nucleoplasty or ozone oxidation for treatment of lumbar disc herniation. Chin J Bone Injury: 672-674, 2014 (In Chinese).

26. Hsu HT, Chang SJ, Yang SS and Chai CL: Learning curve of full-endoscopic lumbar discectomy. Eur Spine J 22: 727-733, 2013.

27. Chaichankul C, Poopitaya S and Tassanawipas W: The effect of learning curve on the results of percutaneous transforaminal endoscopic lumbar discectomy. J Med Assoc Thai 95 (Suppl): S206-S212, 2012.

28. Martín-Láez R, Martínez-Agüeros JA, Suárez-Fernández D, Montiaga-Núñez F and Vázquez-Barquero A: Complications of endoscopic microdiscectomy using the EASYGO! system: Is there any difference with conventional discectomy during the learning-curve period? Acta Neurochir (Wien) 154: 1023-1032, 2012.

29. Nowitzke AM: Assessment of the learning curve for lumbar microendoscopic discectomy. Neurosurgery 56: 755-762, 2005.

30. Gibson JNA, Cowie JG and Iprenburg M: Transforaminal endoscopic spinal surgery: The future 'gold standard' for discectomy?-A review. Surgeon 10: 290-296, 2012.

31. Chen ZG and Fu Q: Percutaneous transforaminal endoscopic discectomy through different approaches for lumbar disc hernation. Zhongguo Gu Shang 25: 1057-1060, 2012 (In Chinese). 